\title{
Automated detection of harm in healthcare with information technology: a systematic review
}

\author{
Malavika Govindan, ${ }^{1}$ Aricca D Van Citters, ${ }^{2}$ Eugene C Nelson, ${ }^{1}$ Jane Kelly-Cummings, ${ }^{3}$ \\ Gautham Suresh ${ }^{4}$
}

- An additional appendix is published online only. To view these files please visit the journal online (http://qshc.bmj. com).

${ }^{1}$ The Dartmouth Institute for Health Policy and Clinical Practice, Center for Leadership and Improvement, Dartmouth Medical School, Lebanon, New Hampshire, USA

${ }^{2}$ Department of Community and Family Medicine, Dartmouth Medical School, Lebanon, New Hampshire, USA

${ }^{3}$ Society for Hospital Medicine, Philadelphia, Pennsylvania, USA ${ }^{4}$ Department of Pediatrics, Dartmouth-Hitchcock Medical

Center, Lebanon, New Hampshire, USA

\section{Correspondence to} Dr Gautham Suresh, Dartmouth-Hitchcock Medical Center, One Medical Center Drive, Lebanon, NH 03756, USA:

gautham.suresh@hitchcock.org

Accepted 14 July 2009

\section{ABSTRACT}

Context To improve patient safety, healthcare facilities are focussing on reducing patient harm. Automated harm-detection methods using information technology show promise for efficiently measuring harm. However, there have been few systematic reviews of their effectiveness.

Objective To perform a systematic literature review to identify, describe and evaluate effectiveness of automated inpatient harm-detection methods.

Methods Data sources included MEDLINE and CINAHL databases indexed through August 2008, extended by bibliographic review and search of citing articles. The authors included articles reporting effectiveness of automated inpatient harm-detection methods, as compared with other detection methods. Two independent reviewers used a standardised abstraction sheet to extract data about automated and comparison harm-detection methods, patient samples and events identified. Differences were resolved by discussion.

Results From 176 articles, 43 articles met inclusion criteria: 39 describing field-defined methods, two using natural language processing and two using both methods. Twenty-one studies used automated methods to detect adverse drug events, 10 detected general adverse events, eight detected nosocomial infections, and four detected other specific adverse events. Compared with gold standard chart review, sensitivity and specificity of automated harm-detection methods ranged from 0.10 to 0.94 and 0.23 to 0.98 , respectively. Studies used heterogeneous methods that often were flawed.

Conclusion Automated methods of harm detection are feasible and some can potentially detect patient harm efficiently. However, effectiveness varied widely, and most studies had methodological weaknesses. More work is needed to develop and assess these tools before they can yield accurate estimates of harm that can be reliably interpreted and compared.

\section{INTRODUCTION}

It is widely recognised that harm caused by the healthcare system is a major source of morbidity and mortality in hospitalised patients. ${ }^{1}$ An estimated 15 million instances of medical harm occur in the USA every year. ${ }^{2}$ However, the lack of simple, practical and accurate methods to identify adverse events in hospitals has hampered efforts to develop routine monitoring systems, assess the impact of interventions to prevent harm and compare interhospital performance.

Detecting incidence and types of patient harm are prerequisites for implementing strategies to prevent harm. Manual, comprehensive chart review by trained professionals has been used in key studies and can be considered the gold-standard harmdetection method. ${ }^{3-6}$ However, this approach requires time and trained abstractors, thereby decreasing its feasibility as a pragmatic method for routine measurement of adverse events.

Several organisations are currently using the Institute for Healthcare Improvement's Global Trigger Tool, which is based on manual chart review, and allows targeted chart review to identify harm more efficiently than comprehensive chart review and more extensively than voluntary reporting of harm.

Automated strategies of harm detection that use computerised methods to scan patient records may require fewer time and personnel resources than traditional methods, and can potentially provide real-time surveillance alerts. We performed this review to: (1) identify types of automated methods of inpatient harm detection described in published literature, (2) describe types of events identified by these methods and (3) evaluate accuracy of these methods in identifying harm. We also independently evaluated the quality and validity of key studies.

\section{METHODS \\ Definitions}

In this review, we used the terms harm, automated harm detection and gold standard chart review as defined in Box 1.

\section{Data sources/study selection}

We (MG and AVC) identified articles for this review through a literature search of MEDLINE (start date 1950) and CINAHL (start date 1982) using the following search terms: (harm OR adverse event OR adverse drug event OR nosocomial infection) AND (automated OR computerised OR electronic) AND (identify OR detect OR detection OR recognise $O R$ recognition). We identified additional articles using bibliographic review of key articles, the 'related articles' feature of Medline, and the 'find similar' and 'find citing articles' feature of CINAHL. We reviewed the title and abstract of each article, and obtained the full text of relevant articles. We limited our search to English language articles indexed through 31 August 2008.

We included studies that: (a) occurred in an inpatient setting, (b) described an automated harmdetection method, (c) measured actual harm and (d) compared the automated method to an alternative method of harm detection. 


\section{Box 1 Definitions}

\section{Harm}

Poor patient outcome resulting from medical care rather than the natural history of the disease, whether or not it was preventable. This term includes adverse medical events (ie, falls, nosocomial infections), adverse drug events and adverse surgical events (ie, postoperative infections, surgical complications). It excludes medical errors that did not result in injury to patients.

\section{Automated harm-detection method}

A method of rapidly searching a large number of patient medical records with a computerised tool to identify actual harm, or indicators (associations) of harm. Records and events identified through computerised screening may then be subjected to further scrutiny by electronic or manual means to verify harm. We defined two degrees of automation: (1) fully automated methods, in which identification of harm was not followed by further chart review, and (2) partially automated methods, in which identified patient records were manually reviewed to verify harm.

\section{Gold standard chart review}

Manual review of the medical record initially by trained personnel, with subsequent review by either a physician or clinical pharmacist to confirm the presence or absence of harm and characteristics of such harm.

\section{Data extraction and analysis}

We developed and tested a standardised data form and extracted the following variables from included articles: details of patient sample, methodology used for automated harm detection, nature of events identified, description of alternative method of harm detection and comparisons of events detected by automated and alternative methods. Data were extracted by MG and AVC, with uncertainties resolved by discussion and consensus.

We critically appraised each study that compared the automated method of harm detection to a gold standard chart review using published criteria for validity of diagnostic test studies. ${ }^{7}$ We assessed each study for: (a) independent, blind comparison of the automated method with a gold standard method, (b) performance of the gold standard assessment regardless of the automated method's results and (c) validation of the assessment in a second, independent set of patients.

If studies provided adequate data, we independently calculated the sensitivity, specificity and positive and negative predictive values of the automated harm-detection method.

\section{RESULTS}

\section{Selection of articles}

One hundred and seventy-six articles were reviewed for potential inclusion, of which 43 provided information on validity of automated methods of harm detection. ${ }^{8-50}$ The remaining articles were excluded because they: were review articles on harm-detection methodologies $(n=9)^{51-59}$; did not focus on detection of harm $(n=26)$ or automated methods $(n=22)$; did not include a comparison group ( $\mathrm{n}=17)$; were not limited to inpatients $(n=13)$; were descriptive papers of a program, incident reporting system, algorithm or computer simulation $(n=33)$; were commentaries or editorials $(n=11)$; or were repeat publications $(\mathrm{n}=2)$.

The methodologies and results from the 43 included studies are described in online appendix 1. Of these, 14 studies compared the automated harm-detection methodology to a gold standard chart review, and their methods and results are summarised in tables 1,2 .

As shown in online appendix 1,20 studies were conducted among adult populations, three in paediatric patients, two among all age groups, one in geriatric patients, one among Medicare beneficiaries and one among patients 14 years and older. The most common hospital settings were general medical units $(n=14)$, followed by general surgical units $(n=8)$, medical, surgical or general intensive care units $(n=8)$, medical subspecialties $(n=3)$, neonatal and paediatric intensive care units $(n=3)$ and obstetric units $(\mathrm{n}=2)$. The target population and setting were unstated in 15 studies.

\section{Data sources for automated harm-detection methods}

Automated harm-detection methods were classified into fielddefined and natural language-processing systems. Field-defined systems relied on computerised detection using pre-existing numeric or coded data stored in medical records. Natural language processing relied on computerised analysis of free text within a medical record to detect language indicative of harm. Field-defined and natural language-processing systems are described in table 3 .

Forty-one of 43 studies used field-defined systems for automated harm detection. The nature of the programs, databases used, data fields used and types of harm detected within this category were source-specific. Typical sources of data for fielddefined programs included laboratory, radiology, microbiology, pharmacy, and administrative and billing databases. Five of 43 studies used natural language-processing systems. The most common source of data was discharge summaries. Radiology reports, chart text, daily progress notes, consultation notes, nursing records, and procedure or operative reports also were used.

\section{Degree of automation}

Twenty-five studies (58\%) reported on detection tools that were partially automated, ${ }^{8-14} 21-25313234-384045-4850 \quad 14$ studies (33\%) described fully automated tools, ${ }^{15-17} 19$ 26-30 3341424449 and one study $(2 \%)$ reported both fully and partially automated systems. ${ }^{20}$ The degree of automation was unclear in three reports $(7 \%) .183943$

\section{Types of events identified}

Automated methods for detecting harm predominantly focused on identification of adverse drug events (ADEs) $(n=21$, 49\%). 111218 21-26 29-32 35-38 434550 Ten automated methods (23\%) focused on general adverse events, ${ }^{8-10} 1933344046-48$ eight (19\%) focused on nosocomial infection, ${ }^{14} 20283941424449$ and four ( $9 \%$ ) focused on other specific adverse events (eg, decubitus ulcers, surgical complications). ${ }^{13} 151727$

\section{Accuracy of automated harm-detection methods}

Only 14 studies $^{15} 1718202223263032-34444748$ compared an automated harm-detection method with 'gold-standard' adverse event detection and were eligible for critical appraisal of validity (table 2). Methodologies used to evaluate these automated systems were heterogeneous. Seven studies $(50 \%)$ applied the gold standard using independent, blind evaluators. Eight studies $(57 \%)$ applied the gold standard independently of the outcome from the automated method. One study $(7 \%)$ validated the results of the automated method in an independent, second set of patients.

Table 4 shows the sensitivity, specificity, and positive and negative predictive values of the automated methods that were 


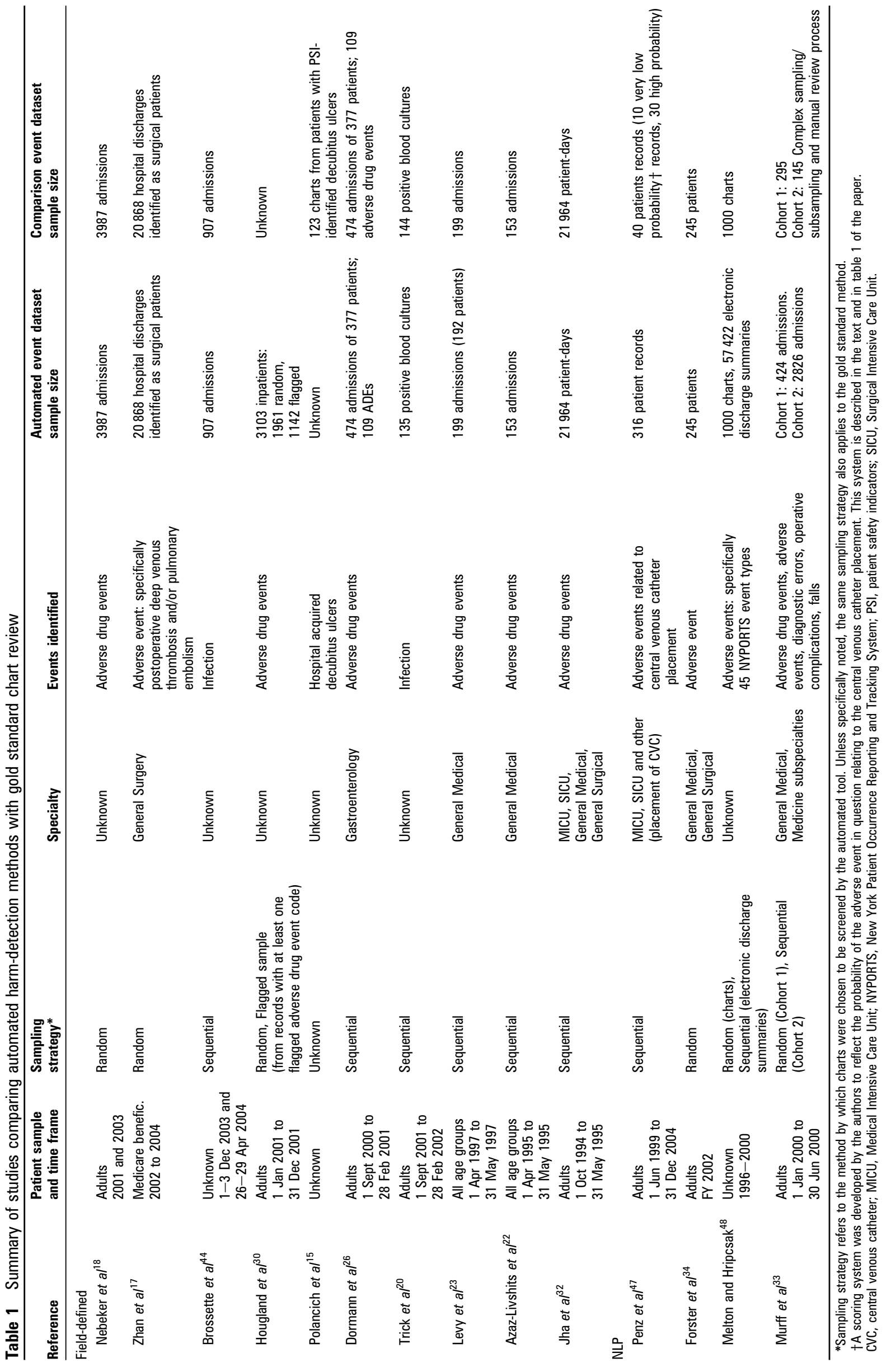




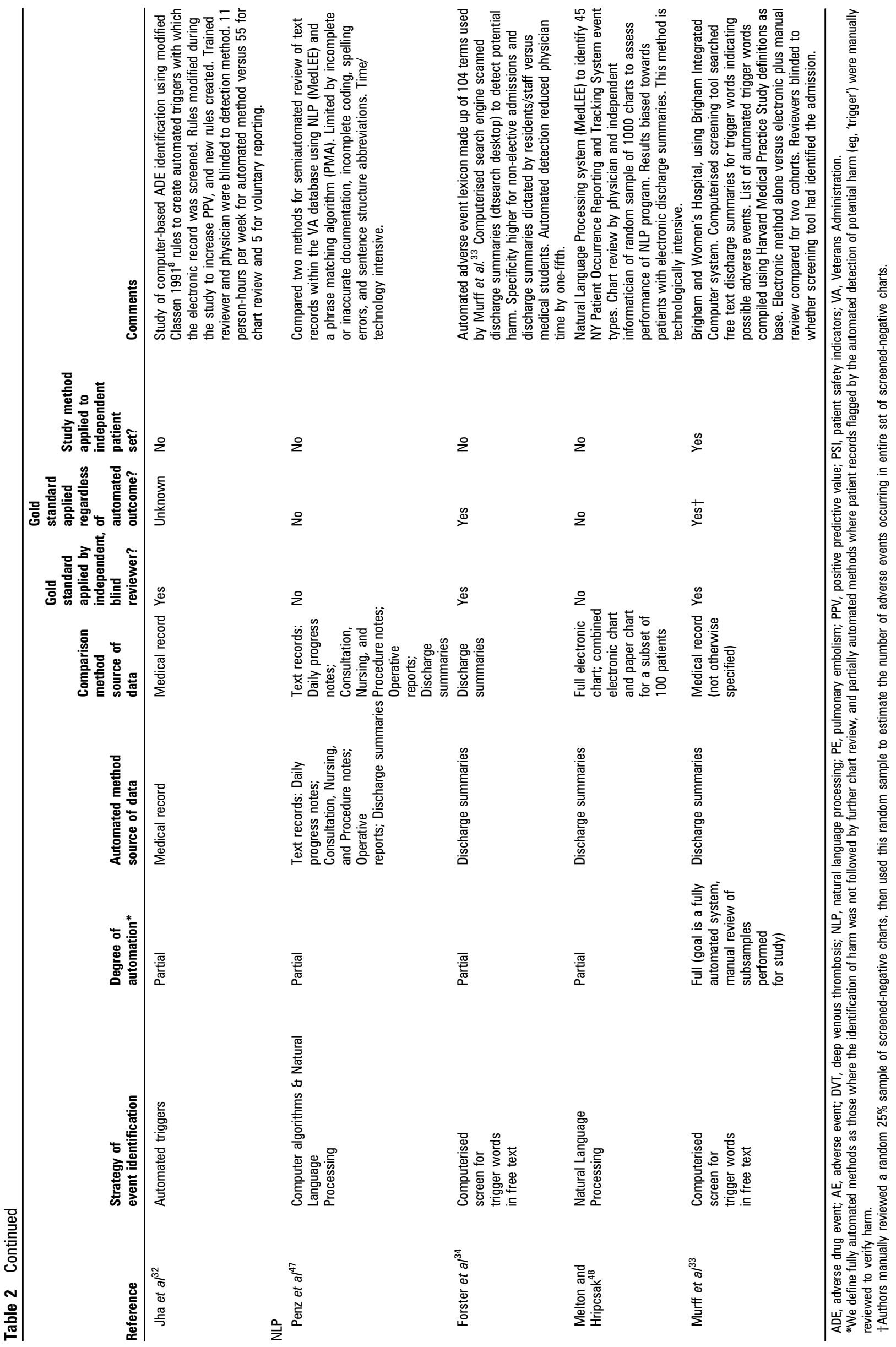




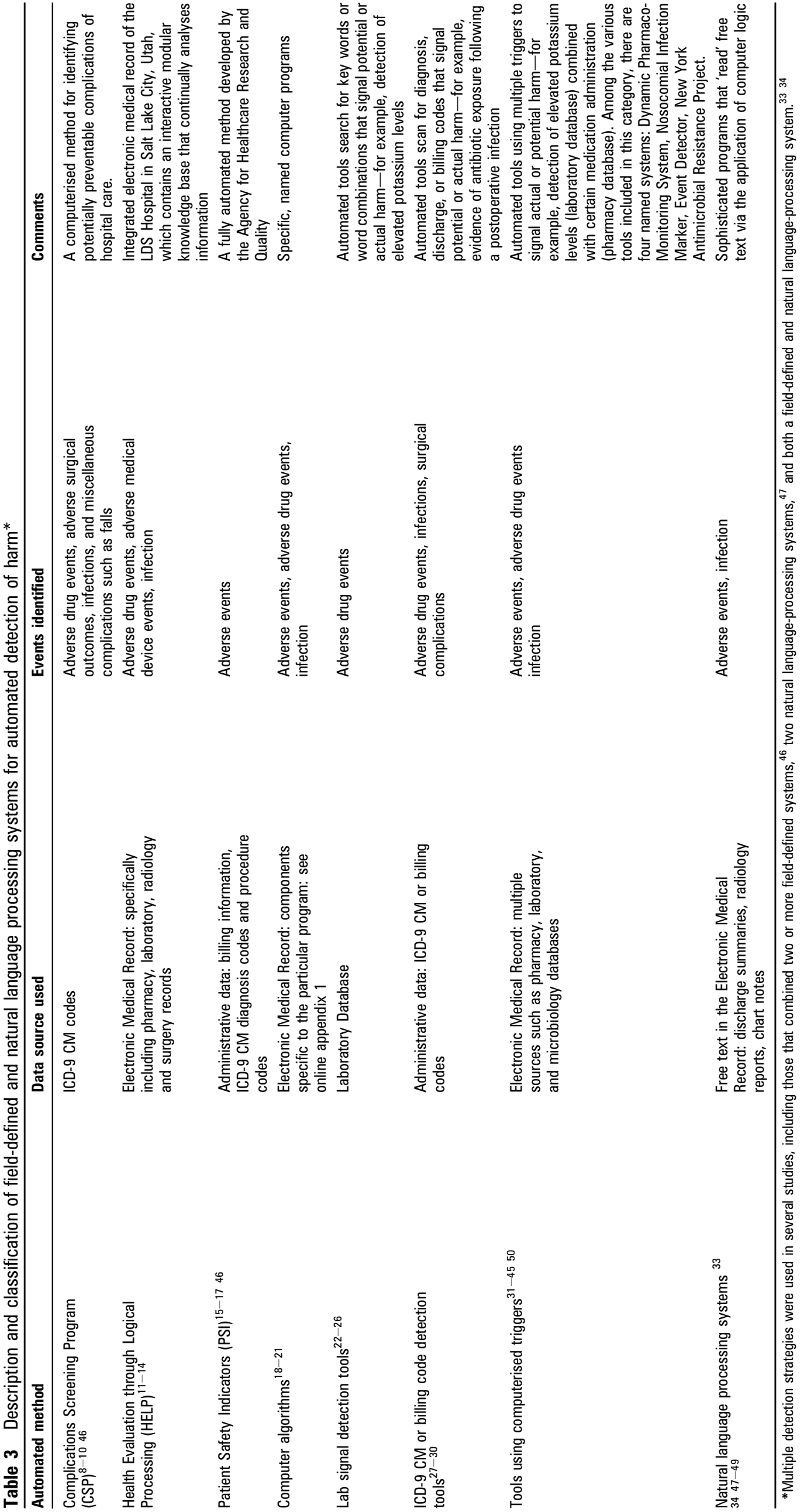




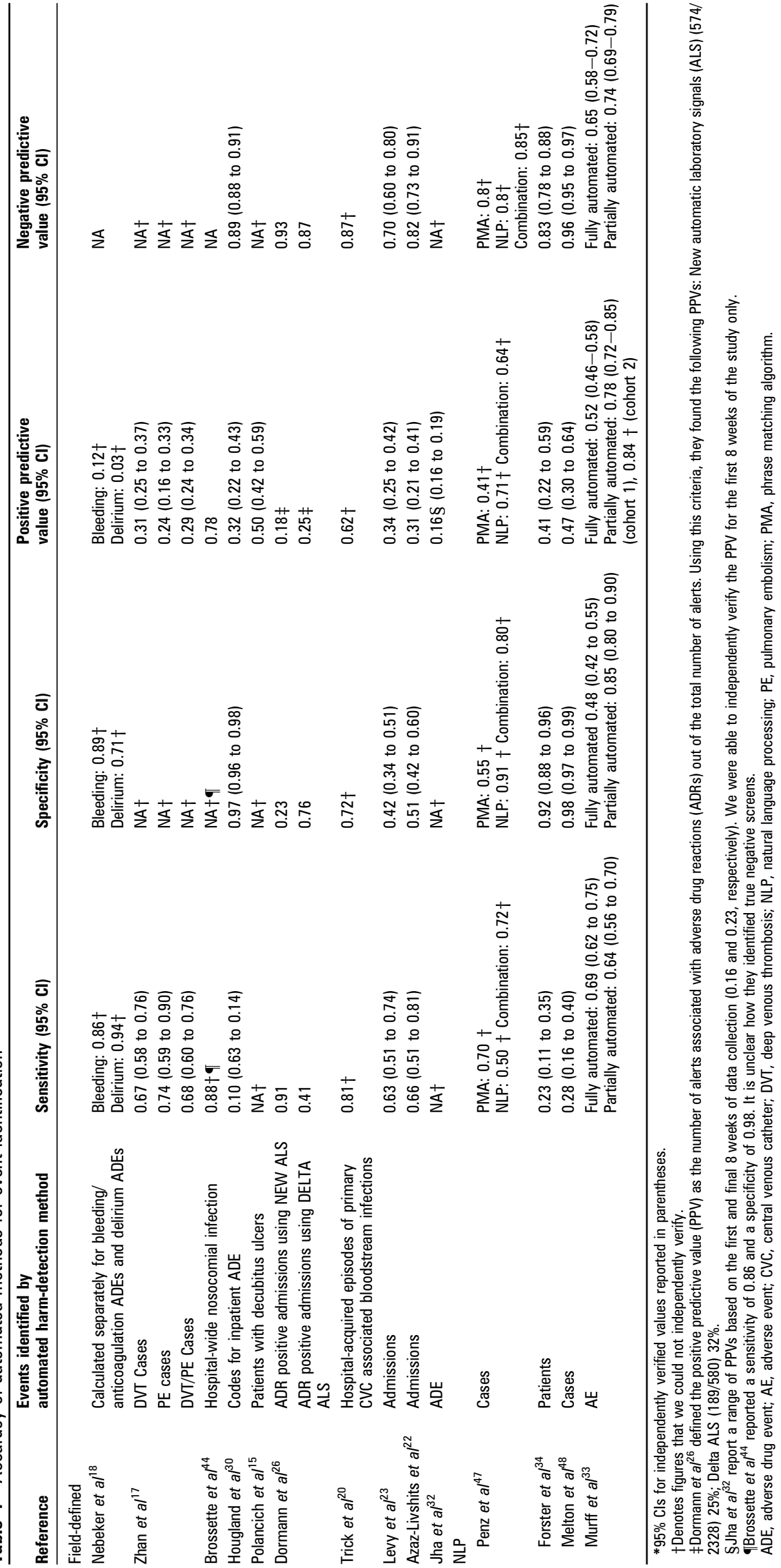


compared against a gold standard chart review. Sensitivities of different methods ranged from 0.10 to 0.94 , and specificities ranged from 0.23 to 0.98 . Positive predictive values ranged from 0.03 to 0.84 , and negative predictive values ranged from 0.70 to 0.96. Our independent assessment of validity allowed us to verify all published values for nine of the 14 studies that reported validity data. ${ }^{15} 1722233033 \quad 3448$ Figure 1 displays the sensitivity and 1-specificity intersection points of methods used in these studies in a format similar to that of a receiver-operating characteristic curve.

\section{DISCUSSION}

Strategies to improve patient safety require efficient and accurate detection of patient harm. Automated methods of harm detection have been used for this purpose because they offer the potential to rapidly scan patient records with minimal human effort. This systematic review describes types of automated methods of harm detection used in inpatient settings, events identified by these methods and their accuracy.

We found two categories of automated harm detection described in the literature: field-defined systems (used in most studies) and natural language-processing systems. Most frequently laboratory, pharmacy and administrative databases were used to identify adverse drug events, general adverse events and nosocomial infections.

We found that the validity of studies describing automated harm-detection methods was variable. Of these studies, those attempting to identify ADEs ${ }^{18} 30$ and nosocomial infections ${ }^{20} 44$ using field-defined methods, and one attempting to identify multiple types of adverse events ${ }^{33}$ using natural language processing satisfied more validity criteria than others. We believe that automated harm-detection methods will have more validity if they attempt to identify events that are discrete, easily and reliably detected, and consistently documented in the chart, such as adverse drug events, nosocomial infections, pressure ulcers and postoperative complications.

Automated harm detection has the potential to positively impact clinical practice. While most automated methods retrospectively identified harm, eight were paired with real-time surveillance alerts that informed physicians or pharmacists of an adverse event. Such prospective surveillance systems can alert the clinical team of impending or ongoing harm, thus allowing early intervention to limit harm. Real-time alerts were present within methods for detecting adverse drug events, ${ }^{11} 2123263545$ general adverse events ${ }^{40}$ and nosocomial infection. ${ }^{14}$ Automated alerts were a component of the Health Evaluation through Logical Processing system ${ }^{11} 14$ and were incorporated within methods using automated lab signal detection, 232645 computer algorithms ${ }^{21}$ and other automated triggers. ${ }^{35} 40$

Another potential benefit of automated detection is the reduction of person-hours required for harm surveillance. Few

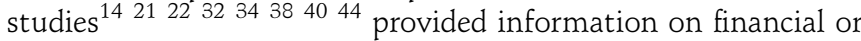
human resource requirements for implementing and maintaining automated detection tools. In general, the automated methods reviewed here require fewer person-hours than manual
Figure 1 Sensitivity by 1-specificity for automated methods compared with gold standard methods of harm detection.

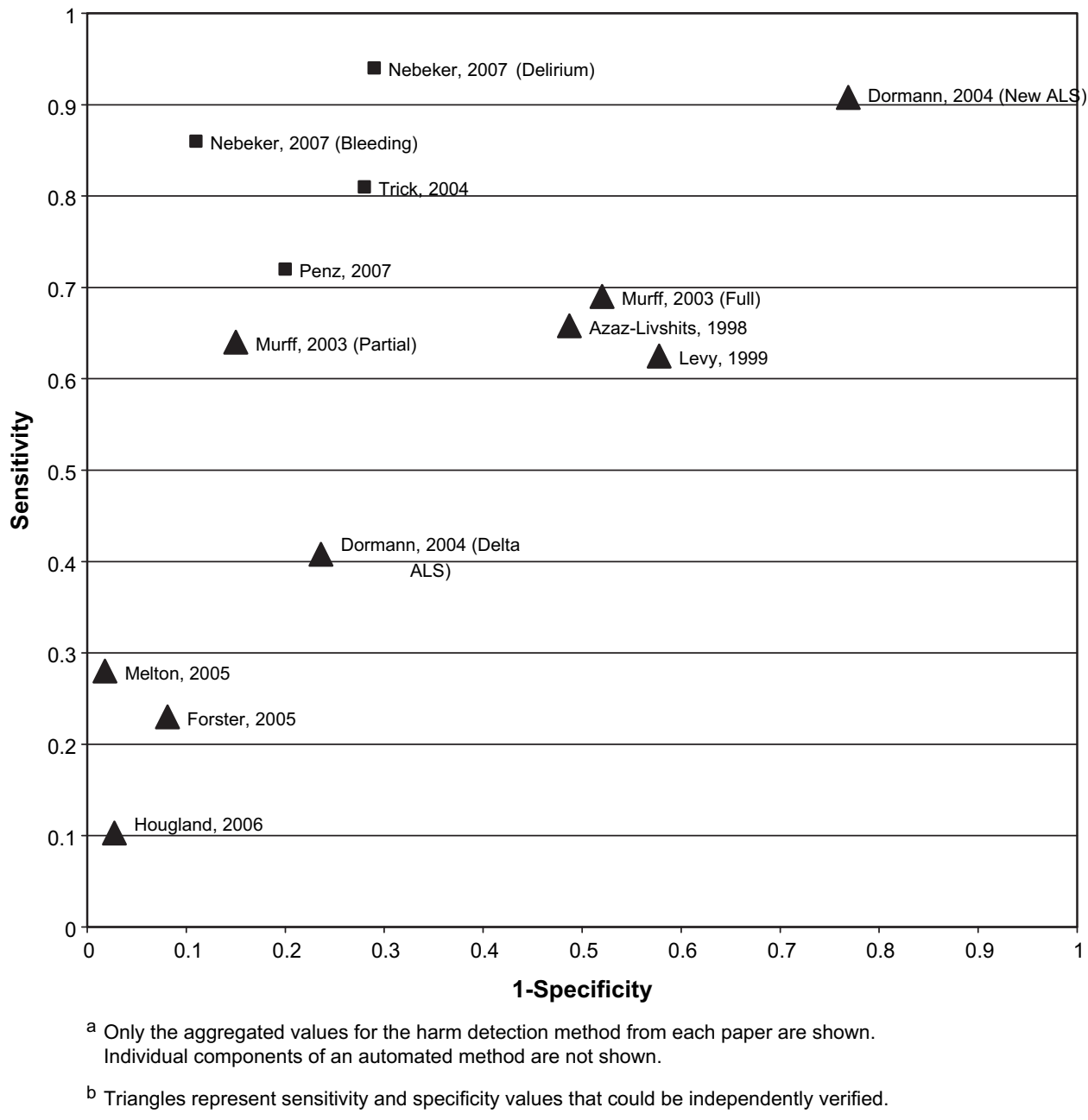


chart review. Field-defined strategies appear to be less technologically demanding than natural language-processing strategies. Sophisticated computer algorithms and natural languageprocessing programs require specialised subject knowledge, skill and time to develop, and require installation and instruction by experts. ${ }^{18} 48$ Whether costs to implement such programs are offset by savings from eliminating manual chart review and decreased patient harm is unknown and should be studied. Future studies also should quantify differences in time and personnel resources needed for the automated detection method, relative to other detection strategies.

To our knowledge, four of the 43 unique articles report on commercially available automated harm-detection systems (MedLEE, ${ }^{48}$ dtsearch desktop, ${ }^{34}$ Nosocomial Infection Marker $(\mathrm{NIM})^{44}$ and Dynamic Pharmaco-Monitoring System ${ }^{45}$ ). Other articles report on systems that employ data elements common across medical institutions (ie, ICD-9 codes used in the Complications Screening Program ${ }^{8-10}$ ) use software available to the VA or specific states (ie, RADARx, NY Antimicrobial Resistance Project $^{2142}$ ) or are available through the Agency for Healthcare Research and Quality (ie, Patient Safety Indicators ${ }^{15-17}$ ). The availability of the remaining detection systems is either institution-specific or not made clear by their developers.

While automated tools offer promise for efficient and accurate harm detection, there are important limitations that currently make them unsuitable for widespread application, particularly for interhospital comparisons. The reported sensitivity and specificity are variable and often low, suggesting that many episodes of harm may go undetected, and that many events identified will be false positives. Low accuracy may result from limited capability of the tool to detect events, or from flawed sources of data used for automated harm detection. For example, the reliability of field-defined systems can be affected by data entry errors or limited availability and accuracy of administrative codes, while natural language processing is sensitive to spelling and grammatical errors in free text. Both systems may include irrelevant or erroneous information, or exclude necessary information. For example, perhaps driven by medical-legal concerns, health professionals often do not include information about medical errors and resulting adverse events in their progress notes, problem lists and discharge summaries. Thus, an electronic medical record containing accurate, complete and easily accessible information can enhance the performance of an automated detection tool. Understanding these factors is important when evaluating the technological requirements, feasibility and inherent limitations of automated detection methods.

The variety of distinct automated methodologies makes comparisons between studies and between automated tools difficult and unreliable. Differences in the quality and content of data sources, as well as other unknowns such as accuracy of hospital documentation and coding practices, also complicate comparisons. The performance and methods of automated tools also may be institution-specific, making it difficult to generalise to other organisations or patient populations. For example, the Health Evaluation through Logical Processing system used by LDS Hospital in Salt Lake City, Utah relies on an advanced, highly integrated and dynamic information system that is not widely available. ${ }^{11-14}$

We speculate that field-defined methods of automated harm detection will prove superior to natural language-processing methods, particularly if information about harm is accurately documented in electronic medical record systems in prespecified fields, thus allowing rapid and reliable detection of harm events.
The methodological rigour of studies was variable. Only twothirds of the 14 studies that compared an automated method with a gold standard chart review had verifiable validity results. Moreover, most studies compared automated harm-detection methods with other sources of data on patient harm (eg, voluntary reporting, ${ }^{11-1324252931373850}$ unstandardised chart reviews, ${ }^{810142836414345}$ and prospective surveillance records ${ }^{4249}$ ). The validity of data from studies without chart review comparison is questionable given the absence of a defined denominator of events against which to measure the performance of the automated tool. The use of different methods, statistical analyses, denominator values and outcomes precludes a comparison of one automated method with another, as well as any attempt to statistically pool their results in a meta-analysis.

Other authors have summarised the literature on automated harm-detection methods, but most have focused on automated methods specific to a type of harm (ie, adverse drug events ${ }^{51} 54$ or nosocomial infections), ${ }^{59}$ patient population (ie, paediatrics), ${ }^{52}$ source of data (ie, administrative data) ${ }^{57}$ or automated technology (ie, natural language processing). ${ }^{58}$ Our systematic review included all types of automated methods, harm events and sources of data evaluated in an inpatient setting. Furthermore, we provide an additional level of critical appraisal compared with other systematic reviews. ${ }^{55} 56$ For example, while Bates et at ${ }^{55}$ address differences between study methodologies by noting the presence or absence of gold standard comparison, they do not assess validity of studies or independently verify reported data. To our knowledge, this is the first systematic review to critically assess methodological rigour and study validity.

While our review has several strengths, it also has limitations. First, the search strategy was limited to published English language articles. Second, we did not evaluate scientific meeting abstracts, nor did we contact investigators to identify unpublished studies. Third, publication bias must be considered in which studies with negative findings may not have reached dissemination venues. Fourth, most of the articles evaluated automated methods of harm detection among adults in general medical or surgical units, which may limit application to other populations and settings. Finally, our independent appraisal of the methodology and validity of key studies relied on information available within published articles. Our inability to verify the rigour and validity of all studies highlights the variation among even the most rigorous evaluations.

In conclusion, our review identified numerous automated methods of harm detection in two broad categories-fielddefined methods and natural language processing-that identified a broad range of harm events, but particularly adverse drug events and nosocomial infections. Although many of these studies described the accuracy (sensitivity and specificity) of automated harm detection when compared with chart review, these results may not be valid due to methodological flaws in the conduct of many of these studies. Future studies assessing the performance of automated harm-detection methods should ensure that the gold-standard assessment (usually chart review) is performed by a blinded assessor, the gold-standard is applied independently of the results of the automated method (ie, charts not flagged by the automated method are reviewed for false negatives), and the automated method is tested in a set of patients that is independent of the set used to develop the automated method. Finally, efforts should be made to improve documentation of harm episodes in the patient record, in problem lists and when generating diagnosis codes, in order to 
improve automated harm detection. Future research should also focus on developing methods for real-time harm detection. In this way, automated harm-detection tools will realise their potential to describe accurately the incidence of harm in hospitalised patients, monitor changes from preventive interventions, and compare institutions and individual health professionals. Establishing universal standards and guidelines for the development, testing and utilisation of automated harmdetection methods, perhaps through a centralised agency, would allow data to be collected and compared in a rigorous, systematic fashion.

\section{Summary}

Automated methods of harm detection are feasible, allow rapid scanning of a large number of patient records with minimal effort and have the potential to identify events as they occur or soon thereafter. However, the heterogeneity of automated methodologies, the spectrum of study rigour and the widely varying accuracy data suggest that currently available automated methods poorly measure the true incidence of harm. These methods cannot replace chart review as the gold standard but can provide estimates of the frequency of harm that can allow hospitals to identify priorities for action, make decisions about safety interventions and potentially monitor change over time. As automated harm-detection tools and scientific methods to test them evolve, there exists a great potential to positively impact patient safety.

Acknowledgements We are grateful for the administrative support provided by the Institute for Healthcare Improvement.

Funding Funding for the literature review was provided by the Institute for Healthcare Improvement (IHI) to MG and ADVC. Subsequent data analysis and interpretation, as well as conceptualisation, preparation, and review of the manuscript were not financially supported.

Competing interests JK-C was employed by Premier Inc. from 31 March 2007 to 2 July 2008. Premier has developed an automated event detection product, SafetySurveillor. This study does not reference or endorse this product. No other authors disclosed any potential conflicts of interest.

Provenance and peer review Not commissioned; externally peer reviewed.

\section{REFERENCES}

1. Institute of Medicine. To err is human. Washington, DC: National Academy Press, 1999 .

2. Institute for Healthcare Improvement. Available at: http://www.IHI.org (accessed 14 Jan 2008)

3. Thomas EJ, Studdert DM, Burstin HR, et al. Incidence and types of adverse events and negligent care in Utah and Colorado. Med Care 2000:38:261-71.

4. Brennan TA, Leape LL, Laird NM, et al. Incidence of adverse events and negligence in hospitalized patients. Results of the Harvard Medical Practice Study I. N Engl J Med 1991:324:370-6.

5. Leape LL, Brennan TA, Laird N, et al. The nature of adverse events in hospitalized patients. Results of the Harvard Medical Practice Study II. N Engl J Med 1991:324:377-84.

6. Wilchesky M, Tamblyn R, Huang A. Validation of diagnostic codes within medical services claims. J Clin Epidemiol 2004:57:131-41.

7. Straus SE, Richardson WS, Glasziou P, et al. Evidence-based medicine: how to practice and teach EBM. 3rd edn. Edinburgh: Churchill Livingstone, 2005.

8. Iezzoni LI, Foley SM, Heeren $\mathrm{T}$, et al. A method for screening the quality of hospital care using administrative data: preliminary validation results. ORB Qual Rev Bull 1992;18:361-71

9. Weingart SN, lezzoni LI, Davis RB, et al. Use of administrative data to find substandard care: validation of the complications screening program. Med Care 2000;38:796-806.

10. Lawthers AG, McCarthy EP, Davis RB, et al. Identification of in-hospital complications from claims data. Is it valid? Med Care2000;38:785-95

11. Classen DC, Pestotnik SL, Evans RS, et al. Computerized surveillance of adverse drug events in hospital patients. JAMA 1991;266:2847-51.

12. Evans RS, Pestotnik SL, Classen DC, et al. Development of a computerized adverse drug event monitor. Proc Annu Symp Comput Appl Med Care 1991:23-7.

13. Samore $\mathbf{M H}$, Evans RS, Lassen A, et al. Surveillance of medical device-related hazards and adverse events in hospitalized patients. JAMA 2004;291:325-34.
14. Evans RS, Larsen RA, Burke JP, et al. Computer surveillance of hospital-acquired infections and antibiotic use. JAMA 1986;256:1007-11.

15. Polancich S, Restrepo E, Prosser J. Cautious use of administrative data for decubitus ulcer outcome reporting. Am J Med Qual 2006;21:262-8.

16. McDonald KM, Romano PS, Geppert J, et al. Measures of Patient Safety Based on Hospital Administrative Data-The Patient Safety Indicators. Technical Review Number 5. (Prepared by the University of California San Francisco-Stanford Evidence-based Practice Center under Contract No. 290-97-0013). AHRO Publication No. 02-0038. Rockville, MD: Agency for Healthcare Research and Quality: August 252002.

17. Zhan C, Battles J, Chiang YP, et al. The validity of ICD-9-CM codes in identifying postoperative deep vein thrombosis and pulmonary embolism. Jt Comm J Qual Patient Saf 2007;33:326-31.

18. Nebeker JR, Yarnold PR, Soltysik RC, et al. Developing indicators of inpatient adverse drug events through nonlinear analysis using administrative data. Med Care 2007:45(10 Suppl 2):S81-8

19. Benson $\mathbf{M}$, Junger $A$, Fuchs $\mathbf{C}$, et al. Using an anesthesia information management system to prove a deficit in voluntary reporting of adverse events in a quality assurance program. J Clin Monit Comput 2000;16:211-17.

20. Trick WE, Zagorski BM, Tokars Jl, et al. Computer algorithms to detect bloodstream infections. Emerg Infect Dis 2004;10:1612-20.

21. Brown S, Black K, Mrochek S, et al. RADARx: recognizing, assessing, and documenting adverse Rx events. Proc AMIA Symp 2000:101-5.

22. Azaz-Livshits T, Levy M, Sadan B, et al. Computerized survelliance of adverse drug reactions in hospital: pilot study. Br J Clin Pharmaco/1998:45:309-14.

23. Levy M, Azaz-Livshits T, Sadan B, et al. Computerized surveillance of adverse drug reactions in hospital: implementation. Eur J Clin Pharmacol 1999;54:887-92.

24. Bagheri $\mathbf{H}$, Michel $\mathrm{F}$, Lapeyre-Mestre $\mathbf{M}$, et al. Detection and incidence of druginduced liver injuries in hospital: a prospective analysis from laboratory signals. $\mathrm{Br} \mathrm{J}$ Clin Pharmacol 2000;50:479-84.

25. Dormann H, Muth-Selbach U, Krebs S, et al. Incidence and costs of adverse drug reactions during hospitalisation: computerised monitoring versus stimulated spontaneous reporting. Drug Saf 2000;22:161-8.

26. Dormann H, Criegee-Rieck M, Neubert $A$, et al. Implementation of a computerassisted monitoring system for the detection of adverse drug reactions in gastroenterology. Aliment Pharmacol Ther 2004;19:303-9.

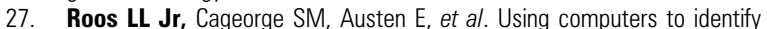
complications after surgery. Am J Public Health 1985;75:1288-95.

28. Hirschhorn LR, Currier JS, Platt R. Electronic surveillance of antibiotic exposure and coded discharge diagnoses as indicators of postoperative infection and other quality assurance measures. Infect Control Hosp Epidemiol 1993;14:21-8.

29. Seeger JD, Schumock GT, Kong SX. Estimating the rate of adverse drug reactions with capture-recapture analysis. Am J Health Syst Pharm 1996;53:178-81.

30. Hougland $\mathbf{P}, \mathrm{Xu}$ W, Pickard $\mathrm{S}$, et al. Performance of international classification of diseases, 9th revision, clinical modification codes as an adverse drug event surveillance system. Med Care 2006;44:629-36.

31. Whipple JK, Quebbeman EJ, Lewis KS, et al. Identification of patient-controlled analgesia overdoses in hospitalized patients: a computerized method of monitoring adverse events. Ann Pharmacother 1994;28:655-8.

32. Jha AK, Kuperman GJ, Teich JM, et al. Identifying adverse drug events: development of a computer-based monitor and comparison with chart review and stimulated voluntary report. J Am Med Inform Assoc 1998;5:305-14.

33. Murff HJ, Forster AJ, Peterson JF, et al. Electronically screening discharge summaries for adverse medical events. J Am Med Inform Assoc 2003; 10:339-50.

34. Forster AJ, Andrade J, van Walraven C. Validation of a discharge summary term search method to detect adverse events. J Am Med Inform Assoc 2005:12:200-6.

35. Hartis CE, Gum MO, Lederer JW Jr. Use of specific indicators to detect warfarinrelated adverse events. Am J Health Syst Pharm 2005;62:1683-8.

36. Mclntosh ST, Petropoulos JB. Using data from automated dispensing units to identify adverse drug reactions. Am J Health Syst Pharm 2005;62:2397-400.

37. Kilbridge PM, Campbell UC, Cozart HB, et al. Automated surveillance for adverse drug events at a community hospital and an academic medical center. J Am Med Inform Assoc 2006;13:372-7.

38. Kilbridge PM, Alexander L, Ahmad A. Implementation of a system for computerized adverse drug event surveillance and intervention at an academic medical center. $J$ Clin Outcomes Manage 2006:13:94-100.

39. Pokorny L, Rovira A, Martin-Baranera M, et al. Automatic detection of patients with nosocomial infection by a computer-based surveillance system: a validation study in a general hospital. Infect Control Hosp Epidemiol 2006;27:500-3.

40. Szekendi MK, Sullivan C, Bobb A, et al. Active surveillance using electronic triggers to detect adverse events in hospitalized patients. Qual Saf Health Care 2006;15:184-90

41. Bellini C, Petignat C, Francioli $P$, et al. Comparison of automated strategies for surveillance of nosocomial bacteremia. Infect Control Hosp Epidemiol 2007;28:1030-5

42. Graham PL 3rd, San Gabriel P, Lutwick S, et al. Validation of a multicenter computer-based surveillance system for hospital-acquired bloodstream infections in neonatal intensive care departments. Am J Infect Control 2004;32:232-4.

43. Huang C, Noirot LA, Reichley RM, et al. Automatic detection of spironolactone-related adverse drug events. AMIA Annu Symp Proc 2005:989. 
44. Brossette SE, Hacek DM, Gavin PJ, et al. A laboratory-based, hospital-wide, electronic marker for nosocomial infection: the future of infection control surveillance? Am J Clin Pathol 2006;125:34-9.

45. Seger AC, Jha AK, Bates DW. Adverse drug event detection in a community hospital utilising computerised medication and laboratory data. Drug Saf 2007; 30:817-24.

46. Weissman JS, Rothschild JM, Bendavid E, et al. Hospital workload and adverse events. Med Care 2007;45:448-55.

47. Penz JF, Wilcox AB, Hurdle JF. Automated identification of adverse events related to central venous catheters. J Biomed Inform 2007;40:174-82.

48. Melton GB, Hripcsak G. Automated detection of adverse events using natural language processing of discharge summaries. J Am Med Inform Assoc 2005; 12:448-57

49. Haas JP, Mendonca EA, Ross B, et al. Use of computerized surveillance to detect nosocomial pneumonia in neonatal intensive care unit patients. Am J Infect Control 2005;33:439-43.

50. Ferranti J, Horvath MM, Cozart $\mathrm{H}$, et al. Reevaluating the safety profile of pediatrics: a comparison of computerized adverse drug event surveillance and voluntary reporting in the pediatric environment. Pediatrics 2008;121:e1201-7.
51. Handler SM, Altman RL, Perera S, et al. A systematic review of the performance characteristics of clinical event monitor signals used to detect adverse drug events in the hospital setting. J Am Med Inform Assoc 2007;14:451-8.

52. Jacobs B. Electronic medical record, error detection, and error reduction: a pediatric critical care perspective. Pediatr Crit Care Med 2007;8(2 Suppl):S17-S20.

53. Chaudhry B, Wang J, Wu S, et al. Systematic review: impact of health information technology on quality, efficiency, and costs of medical care. Ann Intern Med 2006; 144:742-52.

54. Anderson JG. Information technology for detecting medication errors and adverse drug events. Expert Opin Drug Saf 2004;3:449-55.

55. Bates DW, Evans RS, Murff $\mathrm{H}$, et al. Detecting adverse events using information technology. J Am Med Inform Assoc 2003:10:115-28.

56. Murff HJ, Patel VL, Hripcsak G, et al. Detecting adverse events for patient safety research: a review of current methodologies. J Biomed Inform 2003;36:131-43.

57. Zhan C, Miller MR. Administrative data based patient safety research: a critical review. Qual Saf Health Care 2003;12(Suppl 2):iï8-63.

58. Spyns P. Natural language processing in medicine: an overview. Methods Inf Med 1996;35:285-301.

59. Leal J, Laupland KB. Validity of electronic surveillance systems: a systematic review. $\checkmark$ Hosp Infect 2008;69:220-9. 